\title{
GiLAN Roaming: Roam Like at Home in a Multi-Provider NFV Environment
}

\author{
Juhoon Kim $^{1}$, Luis M. Contreras ${ }^{2,4}$, Pierluigi Greto ${ }^{3}$, Hilmar Magnusson ${ }^{3}$ \\ Hagen Woesner ${ }^{3}$, Daniel Fritzsche ${ }^{3}$, Luca Cominardi ${ }^{4}$, Carlos J. Bernardos ${ }^{4}$ \\ ${ }^{1}$ Deutsche Telekom AG, ${ }^{2}$ Telefonica I+D, ${ }^{3}$ BISDN GmbH, ${ }^{4}$ University Carlos III of Madrid
}

\begin{abstract}
Roam Like at Home' is a slogan used to express the new roaming regulation applied to the countries within the European Union. Since June 15th, 2017, mobile network subscribers of the network operators within the European Union can use mobile services in other European countries without having extra charges for it. Although almost all mobile subscribers within countries of the European Union benefits from the new roaming rule, many mobile service providers are yet to be adapted to this change. Therefore, those providers bear the burden of the cost for the inter-provider traffic. Moreover, the latency between subscribers and their operator specific content must be reduced in order to provide a roaming service 'like at home'. An emerging virtualization technology such as a Network Function Virtualization (NFV) sheds light on these challenges.

This paper describes the concept of the mobile roaming service and our on-going implementation work as a part of 5G-PPP 5GEx project which aims at localizing inter-provider mobile traffic within the virtualized network infrastructure.
\end{abstract}

\section{INTRODUCTION}

Subscribers of a mobile network may access their telecommunication service by using mobile devices in different networks or even in different countries. This type of inter-provider services is commonly known as an International Mobile Roaming (IMR) and it typically involves high costs compared to the domestic service usage. Therefore, subscribers are cautious about their usage of mobile services (e.g., data transfer, voice call and SMS) while travelling to other countries. This greatly limits the convenience of utilizing today's telecommunication infrastructure. The European Union (EU) perceives expensive roaming charges between member states as a barrier to an economic alliance. This view led to the agreement of abolishing roaming charges within the European Union under the slogan of 'Roam Like at Home'.

The new roaming regulation applies to 28 countries within the European Union starting from June 15th, 2017. However, the mobile service operators provide their roaming services on the same infrastructure as before, thus posing two problems: $i$ ) mobile service providers are fully responsible for the operation cost (OPEX) of the inter-provider traffic (e.g., consumption of home operator specific content) and it turns into unexpected expenses, and ii) when roaming service users access the home operator specific services, data traffic must be routed from/to the home network and it causes a significant amount of delays for the mobile subscribers which does not suit its slogan (i.e., 'Roam Like at Home').

978-1-5386-3779-1/18/\$31.00 (c)2018 IEEE
The latest trend in network virtualization creates a new possibility for these subjects. More precisely, Network Function Virtualization (NFV) enables mobile network operators to modularize existing physical network entities in a virtualized system environment. In particular, Sahai et al. [1] showed the possibility of virtualizing network functions between Packet Data Network Gateway (PGW) and Packet Data Network (PDN).

Thanks to recent virtualization efforts made by developers of Evolved Packet Systems (e.g., OpenEPC), we conduct an experiment of virtualizing a home network service in a visited network. The experiment is performed on a multi-provider NFV platform which is currently developed as a part of the 5G-Exchange (5GEx) project [2]. In this paper, we describe the general concept of 5GEx architecture and our experiment on the virtualization of mobile roaming service as a Slice-asa-Service (SaaS) example.

The rest of this paper is structured as follows. Section II and Section III give an overview of today's roaming architecture and a brief description of NFV and 5GEx architecture, respectively. Section IV describes the process and method of home service virtualization in our service architecture. In Section $\mathrm{V}$, remaining challenges of the GiLAN roaming service are discussed. Section VI summarizes related studies and standardization efforts and Section VII concludes this paper.

\section{Roaming in Today's Mobile Network}

This section describes the architecture of the inter-provider service roaming in today's mobile network system.

The international roaming agreements established among Mobile Network Operators (MNOs) are the main enablers for the IMR service. Such agreements imply i) a number of control plane transactions among the packet core entities of each network and ii) monetary compensation for facilitating the usage of the visited network. In case of not having direct interconnection at data plane, intermediate transit networks and Internetwork Packet Exchange (IPX) [3] are needed for delivering data between operators, thus enabling end-to-end connectivity.

Figure 1 depicts a simplified view of the architecture for mobile roaming between two domains in today's Evolved Packet System (EPS). The roaming procedure is triggered when a mobile subscriber (UE) of a mobile network (i.e., 


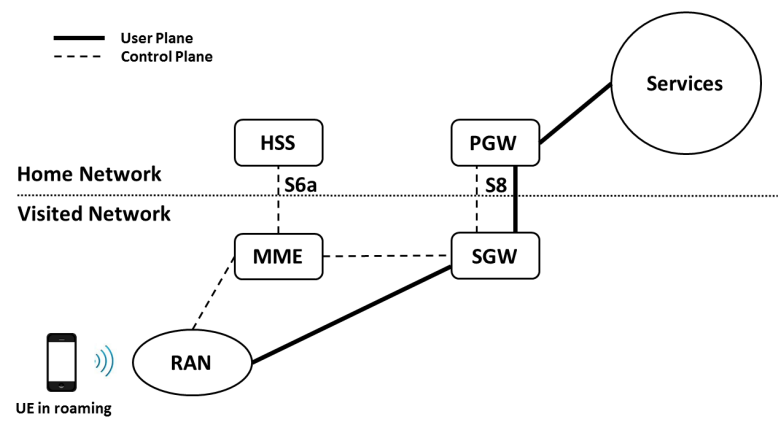

Fig. 1: A simplified architecture of an Evolved Packet System

home network) travels to a foreign network under a different administration (i.e., visited network), attempting to join his/her services by using the Radio Access Network (RAN) infrastructure of the visited network.

The MME (Mobility Management Entity) of the visited network identifies the newly connected device and tries to register it into the system. During this process, the MME detects that the UE belongs to another network. In case that the two operators have an IMR agreement in place, the MME of the visited network retrieves the information of the subscriber's UE from the Home Subscriber Server (HSS) of the home network. With such information, the UE appears in the home HSS as located in the visited network and the roaming subscriber can use his/her home network services from the visited network infrastructure.

The roaming traffic can be categorized as control plane traffic and data (or user) plane traffic. The control plane traffic includes the subscriber information and charging information and it has typically a small footprint on the exchanged data as opposed to data plane traffic. When the subscriber uses a specific service of his/her home operator or accesses external networks, the data plane traffic needs to be always routed to the home network through the home PGW [4]. The S8 interface identifies the logical connection between SGW (Serving Gateway) and PGW in the EPS as shown in Figure 1. In roaming scenarios, the S8 interface connects SGW and PGW nodes from different administrative domains, thus allowing the users to roam from a visited network to a home network and vice versa.

\section{NFV AND 5GEX}

This section provides an overview on Network Function Virtualization (NFV) and introduces the 5GEx architecture as our target platform.

NFV enables the instantiation of Virtual Network Functions (VNFs) on commodity hardware at different locations, thus allowing to scale the network in/out and up/down according to the real service demand. The dedicated and specialized hardware is substituted by computing elements (typically x 86 servers) with a virtualization layer (hypervisors) which execute in software as the same kind of a service function supported by the traditional specialized network equipment. The set of those computing resources is typically referred to as Network Function Virtualization Infrastructure (NFVI), which provides various Points of Presence (NFVI-PoPs).

In initial ETSI NFV concepts, such PoPs were intended to be under a single provider administration. However, the extension of the architectural framework of network virtualization to cover multiple administrative domains is being investigated [5]. In such scenarios, service providers can offer their NFVI-PoPs to host third party service functions or even offer VNFs to be consumed by others. As a result, VNFs can be instantiated in different administrative domains and multiple geographical locations, thus decoupling the service from the hosting infrastructure and from the administrative domain.

In this direction, the 5GEx project is developing a network orchestration ecosystem [6] enabling the trading of network functions and resources in a multi-provider environment, targeting a Slice-as-a-Service approach. Figure 2 presents a high-level overview of the 5GEx architecture. Different providers participate at this ecosystem, each of them representing a distinct administrative domain interworking through Multi-domain Orchestrators (MdOs) for the provision of services in a multi-provider environment. This architecture extends the ETSI MANO NFV management and orchestration framework [7] for facilitating the orchestration of services across multiple administrative domains, which may belong to different infrastructure operators or service providers. Each $\mathrm{MdO}$ handles the orchestration of resources and services from different providers, coordinating resource and/or service orchestration at multi-provider level, orchestrating resources and/or services using Domain Orchestrators belonging to each of the multiple administrative domains.

The Domain Orchestrators are in charge of performing Service Orchestration and/or Resource Orchestration leveraging on the abstractions exposed by the underlying Resource Domains, which cover a variety of technologies. There are three main interworking interfaces and APIs identified in the 5GEx architecture framework. The $\mathrm{MdO}$ exposes service specification APIs (Customer-to-Business, C2B) that allow business customers to specify their requirements for a service on interface I1. The MdO interacts with other MdOs via interface I2 (Business-to-Business, B2B) to request and orchestrate resources and services across administrative domains. Finally, the MdO interacts with Domain Orchestrators via interface I3 APIs to orchestrate resources and services within the same administrative domains.

\section{GiLAN ROAMING}

This section provides a description of the GiLAN roaming currently developed as one of Slice-as-a-Service (SaaS) examples on the 5GEx architecture. The goals of the GiLAN roaming service are $i$ ) to reduce the OPEX by localizing data traffic within the visited network and ii) to reduce the latency between users and service specific content. 


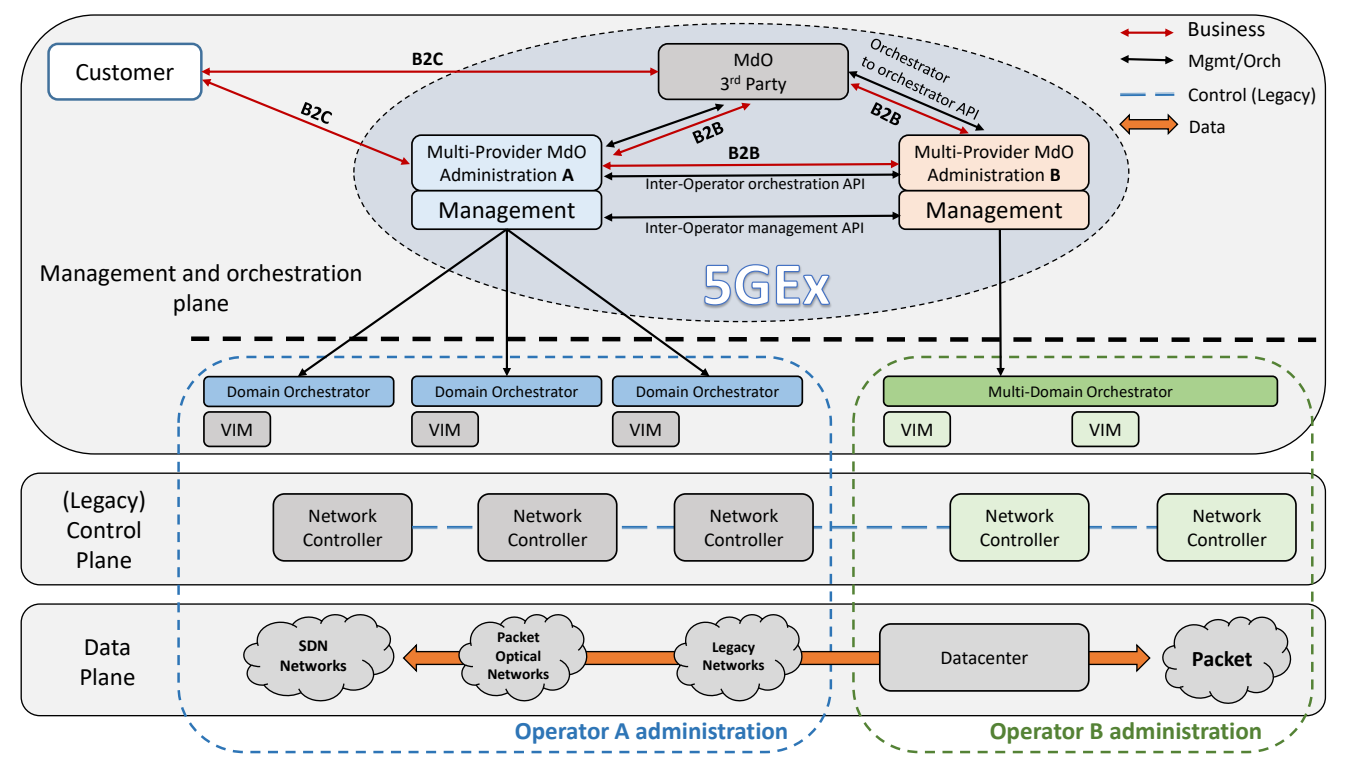

Fig. 2: 5GEx architectural framework

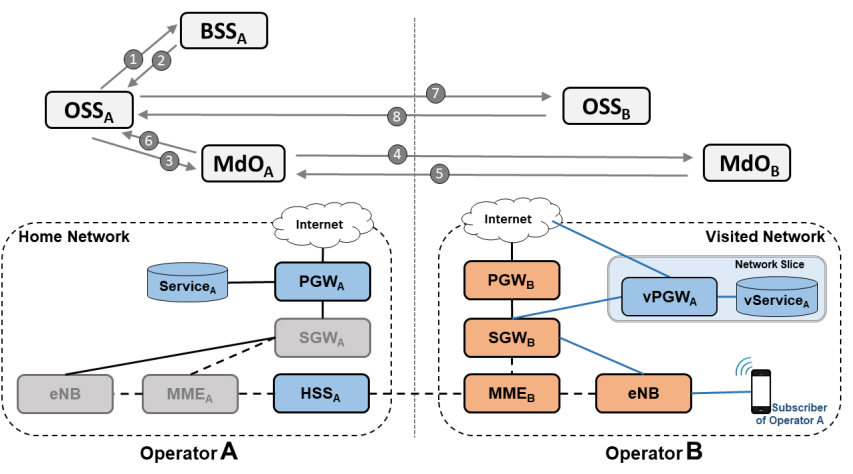

Fig. 3: GiLAN roaming scenario on the 5GEx architecture

\section{A. Deployment of the GiLAN roaming service}

The procedure of the GiLAN roaming service creation can be broken down into three different phases. In the first phase, 5GEx MdOs exchange their service capabilities with other MdOs via the B2B interface (I2, see Section III). As a result of this exchange, the list of the available services of the federated operators is published in the form of a service catalogue on each MdO.

The second phase includes the interaction between operator and $\mathrm{MdO}$ via the $\mathrm{C} 2 \mathrm{~B}$ interface (I1, see Section III and Figure 2). This interaction starts a negotiation process between different operators and their MdOs (via I2 interface). At this point, the operator willing to initiate the service in another network is aware of the capabilities of the target network. Figure 3 illustrates the general interactions between operators and required VNFs and connectivity in the infrastructure.

Numbered lines in Figure 3 depict the detailed interactions between the two operators and their MdOs to setup a GiLAN roaming service in the second phase. These processes are described below:

1) $O S S_{A}$ notifies that the threshold on a number of users roaming in Operator $_{B}$ 's network (i.e., visited network) is exceeded;

2) $B S S_{A}$ instructs the $O S S_{A}$ to deploy the GiLAN roaming service in the visited network;

3) $O S S_{A}$ instructs the $M d O_{A}$ to deploy the VNFs and to establish the required connectivity in the visited network;

4) $M d O_{A}$ requests $M d O_{B}$ to deploy vService $_{A}$ (i.e., virtualized service of Operator $_{A}$ ) and $v P G W_{A}$ (i.e., virtualized PGW of Operator $_{A}$ ) in the visited network;

5) $M d O_{B}$ notifies the success of the deployment to $M d O_{A}$ with interface IDs of the deployed VNFs;

6) $M d O_{A}$ forwards the information to $O S S_{A} . O S S_{A}$ is able to monitor and configure specific VNFs deployed in the visited network by using the interface IDs;

7) To complete the service establishment, $O S S_{A}$ requests $O S S_{B}$ to configure appropriate network functions which are under the administration of Operator $_{B}$;

8) $O S S_{B}$ notifies success at configuring its own VNFs.

Finally, in the third phase, the roaming UE discovers the $v P G W_{A}$ which in turn grants access to the user. At this point, the user can use the $v P G W_{A}$ instead of the PGW of Operator $_{B}$. However, such a decision is made by a network function of Operator $_{B}$, which requires prior configuration, as reported in step 7) of the second phase. The next section elaborates on how the mobile device of a roaming user discovers $v P G W_{A}$ in the visited network.

\section{B. Discovery of the virtualized $P G W$}

As briefly explained in Section II, when a UE (a subscriber of Operator $_{A}$ ) sends an attachment request to the Operator $_{B}$ 's network, Operator $B$ 's MME identifies the 
roaming UE and registers the UE into the visited network based on the information acquired from the home network's HSS. During this process, the HSS provides an identifier of a PGW (PGW-ID) for the MME which the UE must connect to. Typically, a PGW-ID has the form of a Fully Qualified Domain Name (FQDN) and this FQDN is translated into an IP address by a DNS server within the visited network.

To accomplish the connectivity for the GiLAN roaming, the home HSS $\left(H S S_{A}\right)$ provides an FQDN of $v P G W_{A}$ to the MME. Since a mapping between the FQDN and the IP address of the $v P G W_{A}$ is registered to the database of the DNS server during the service creation of the GiLAN roaming (see the previous section), the UE can connect to $v P G W_{A}$ and access the service via this virtualized PGW.

\section{Discussion}

In this paper, we presented the concept of the GiLAN roaming service which focuses on localizing the inter-provider mobile roaming traffic in virtualized network environments. Nonetheless, there are several unanswered questions from technical, operation, and business perspectives which we plan to address as on-going work.

For example, methods for introducing and/or modifying a service near real time is yet to be devised. Assuring the Quality of Service (QoS) such as the latency level and bandwidth in a foreign network infrastructure is another difficulty to cope with.

Moreover, due to the increasing demand for a high bandwidth, the development of cost cutting strategies for leasing network resources became of paramount importance. This also brings up the necessity of an appropriate charging model between operators.

In case of cloud-based services, the user's data may need to be duplicated in the visited network and thus security and privacy concerns may arouse. Therefore, a strong encryption policy must be designed and applied to such services.

\section{RELATED WORK}

In this section, we provide an overview of relevant standardization activities and literature related to our work.

The concept of the GiLAN roaming service is based on the existing LTE mobile network architecture (i. e., Evolved Packet System) because the next generation mobile network $(5 \mathrm{G})$ is still being defined. However, we observe $5 \mathrm{G}$ standardization activities in order to align with the aim of the 5GEx project. In particular, we pay a sharp attention to the on-going 3GPP specifications (e.g., TS 23.501 [8]) in which the 5G system architecture (including the roaming architecture) is actively debated. According to the current status of the specification, the roaming scenarios remain the same (i.e., a local breakout scenario and a home routed scenario). Therefore, we believe that the results of our work are influential for the 5G-enabled mobile network.

J. Erfanian et al. [9] refers to the global roaming scenario in wireless networks as an important example of a multi domain NFV architecture. More precisely, the white paper cites an example of deploying a VNF which supports the home service in a visited network by acquiring a network slice from the visited network. They do not provide a specific architecture of the roaming scenario, but the idea aligns with the concept of GiLAN roaming.

Sahai et al. [1] introduces the virtualization architecture of network functions on SGi/Gi reference points. Although the architecture does not concern a roaming situation and the technical details of the virtualization may differ from the one used in our approach, their work and demonstrations provide the fundamental concepts of the GiLAN roaming in a virtualized network environment.

\section{CONCLUSION AND Future WORK}

In this paper, we presented the concept of the GiLAN roaming which aims at localizing inter-provider network traffic in the visited network with the help of the emerging network virtualization technology. For a better understanding of the GiLAN roaming concept, we described the architecture of the 5GEx multi-provider NFV system on which the GiLAN roaming test-bed is built.

Future work will focus on verification and performance evaluation of our concept including the assessment of traffic reduction between different administrative domains as well as the latency improvement of roaming services in content delivery.

\section{ACKNOWLEDGEMENT}

This work has been performed in the framework of the H2020-ICT-2014 project 5GEx (Grant Agreement no. 671636), which is partly funded by the European Commission. This information reflects the consortium view, but neither the consortium nor the European Commission are liable for any use that may be done of the information contained therein.

We would also like to show our gratitude to Core Network Dynamics for allowing us to use their product (OpenEPC) during the course of our research and for supporting us by sharing their knowledge.

\section{REFERENCES}

[1] A. Sahai, P. Grnsund, J. Guesman, S. Bajaj, N. Lemieux, S. Kilaru, "Subscriber Aware SGi/Gi-LAN Virtualization," 2014.

[2] "A 5GEx White Paper: 5GEx Multi-domain Service Creation," March 2016.

[3] GSMA Association IR.34, "Inter-Service Provider IP Backbone Guidelines, version 13.0," October 2016.

[4] GSMA Association, "LTE and EPC Roaming Guidelines, version 15.0," November 2016.

[5] C.J. Bernardos, L.M. Contreras, I. Vaishnavi, R. Szabo, "Multi-Domain Network Virtualization, draft-bernardos-nfvrg-multidomain-02," March 2017.

[6] C. J. Bernardos, B. Ger, M. Di Girolamo, A. Kern, B. Martini, I. Vaishnavi, "5GEx: Realizing a Europe Wide Multi-Domain Framework for Software Defined Infrastrcutures," in Transactions on Emerging Telecommunications Technologies, Vol. 27, Issue 9, pp. 1271-1280, September 2016.

[7] "ETSI NFV ISG, Networks Functions Virtualization (NFV), Management and Orchestration, ETSI GS NFV MAN 001, V1.1.1," December 2014.

[8] "3GPP TS 23.501: System Architecture for the 5G System; Stage 2 (Release 15), work in progress," March 2017.

[9] J. Erfanian et al., "Network Functions Virtualisation - White Paper on NFV priorities for 5G," February 2017. 